\title{
Prioritized Expression of BTN2 of Saccharomyces cerevisiae under Pronounced Translation Repression Induced by Severe Ethanol Stress
}

\author{
Yukina Yamauchi and Shingo Izawa* \\ Laboratory of Microbial Technology, Department of Applied Biology, Graduate School of Science and Technology, Kyoto \\ Institute of Technology, Kyoto, Japan
}

OPEN ACCESS

Edited by:

Weiwen Zhang,

Tianjin University, China

Reviewed by:

Xueyang Feng,

Virginia Tech, USA

Daisuke Watanabe,

Nara Institute of Science

and Technology, Japan

*Correspondence:

Shingo Izawa

thioredoxin@kit.ac.jp

Specialty section:

This article was submitted to Microbiotechnology, Ecotoxicology

and Bioremediation,

a section of the journal

Frontiers in Microbiology

Received: 04 July 2016 Accepted: 10 August 2016

Published: 23 August 2016

Citation:

Yamauchi Y and Izawa S (2016)

Prioritized Expression of BTN2 of Saccharomyces cerevisiae under Pronounced Translation Repression Induced by Severe Ethanol Stress.

Front. Microbiol. 7:1319.

doi: 10.3389/fmicb.2016.01319
Severe ethanol stress ( $>9 \%$ ethanol, $v / v$ ) as well as glucose deprivation rapidly induces a pronounced repression of overall protein synthesis in budding yeast Saccharomyces cerevisiae. Therefore, transcriptional activation in yeast cells under severe ethanol stress does not always indicate the production of expected protein levels. Messenger RNAs of genes containing heat shock elements can be intensively translated under glucose deprivation, suggesting that some mRNAs are preferentially translated even under severe ethanol stress. In the present study, we tried to identify the mRNA that can be preferentially translated under severe ethanol stress. BTN2 encodes a v-SNARE binding protein, and its null mutant shows hypersensitivity to ethanol. We found that BTN2 mRNA was efficiently translated under severe ethanol stress but not under mild ethanol stress. Moreover, the increased Btn2 protein levels caused by severe ethanol stress were smoothly decreased with the elimination of ethanol stress. These findings suggested that severe ethanol stress extensively induced BTN2 expression. Further, the BTN2 promoter induced protein synthesis of non-native genes such as CUR1, GIC2, and YUR1 in the presence of high ethanol concentrations, indicating that this promoter overcame severe ethanol stress-induced translation repression. Thus, our findings provide an important clue about yeast response to severe ethanol stress and suggest that the BTN2 promoter can be used to improve the efficiency of ethanol production and stress tolerance of yeast cells by modifying gene expression in the presence of high ethanol concentration.

Keywords: Saccharomyces cerevisiae, ethanol stress, translation repression, alcoholic fermentation, preferential translation, BTN2

\section{INTRODUCTION}

Budding yeast Saccharomyces cerevisiae produces ethanol through alcoholic fermentation. Ethanol concentrations in wine must and Japanese sake mash reach high levels in the final stage of brewing. High ethanol concentration exerts adverse effects on yeast cells and inhibits yeast cell growth and viability by inducing severe stress. Ethanol concentration of $>9 \%(\mathrm{v} / \mathrm{v})$ blocks the nuclear export of bulk poly $(\mathrm{A})^{+}$mRNA and represses translation initiation in yeast cells (Takemura et al., 2004; Izawa et al., 2005a,b; Iwaki et al., 2013; Yamamoto and Izawa, 2013). Repression of overall protein synthesis in yeast cells under severe ethanol stress indicates that increased mRNA expression does 
not always result in the expected increase in protein expression (Izawa, 2010, 2015). Pronounced repression of overall protein synthesis seems to be one of the primary causes of growth suppression of yeast cells under severe ethanol stress.

During translation repression, untranslated mRNAs leave the translation apparatus and form the cytoplasmic messenger ribonucleoprotein ( $\mathrm{mRNP}$ ) granules such as processing bodies (P-bodies) and stress granules (SGs) under severe stress conditions. It has been reported that glucose deprivation, $\mathrm{NaN}_{3}$, high vanillin concentration, and robust heat shock repress translation activity in yeast cells and induce the formation of P-bodies and SGs (Teixeira et al., 2005; Balagopal and Parker, 2009; Buchan and Parker, 2009; Grousl et al., 2009; Buchan et al., 2011; Nguyen et al., 2014, 2015). P-bodies and SGs play important roles in the regulation of gene expression under severe stress (Balagopal and Parker, 2009; Buchan and Parker, 2009). Severe ethanol stress also activates the formation of P-bodies and SGs in yeast cells (Izawa et al., 2007; Kato et al., 2011).

Proteins required for stress tolerance are intensively synthesized under severe stress despite the pronounced repression of translation activity. Glucose deprivation rapidly causes a reduction in overall protein synthesis in yeast cells (Ashe et al., 2000). Zid and O'Shea (2014) reported that mRNAs of genes encoding small heat shock proteins (sHSPs), such as HSP26 and HSP30, are preferentially translated during glucose deprivation. Promoter sequences of these genes contain heat shock elements (HSEs) that not only affect the mRNA levels of these genes but also affect the efficiency of mRNA translation during glucose deprivation. Recently, we also reported that the $B D H 2$ promoter and $A D H 7$ promoter-driven mRNAs were preferentially translated under severe vanillin stress, which induces translation repression (Nguyen et al., 2015; Ishida et al., 2016). However, no information is available on yeast mRNAs that are efficiently translated under severe ethanol stress. Identification of these mRNAs is important for understanding the response of yeast cells to severe stress.

Therefore, we examined the expression of previously reported genes associated with ethanol tolerance. In the present study, we focused on BTN2 whose deficiency induces hypersensitivity to ethanol (Espinazo-Romeu et al., 2008; Yang et al., 2011). BTN2 encodes a v-SNARE binding protein that is involved in intracellular protein trafficking (Kama et al., 2007) and plays a role in protein deposition in the nucleus (Miller et al., 2015). Because Btn2 is important for the correct localization of various proteins, btn $2 \Delta$ cells show pleiotropic phenotypes, including decreased resistance to ethanol, acidic $\mathrm{pH}$, hydrostatic pressure, and L-canavanine (Chattopadhyay et al., 2000; Chattopadhyay and Pearce, 2002; Kim et al., 2005; Espinazo-Romeu et al., 2008; Yang et al., 2011). We found that BTN2 mRNA was efficiently translated under severe ethanol stress and that Btn2 protein levels decreased after ethanol elimination. Moreover, the BTN2 promoter induced the expression of non-native genes such as CUR1, GIC2, and YUR1 under severe ethanol stress. These findings suggested that BTN2 expression responded to severe ethanol stress and that the BTN2 promoter could be used to improve ethanol tolerance or produce useful proteins during brewing by modifying yeast gene expression under severe ethanol stress.

\section{MATERIALS AND METHODS}

\section{Strains and Medium}

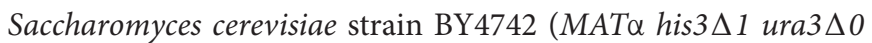
leu $2 \Delta 0$ lys $2 \Delta 0$ ) was used in this study. Yeast cells were cultured in SD medium ( $2 \%$ glucose, $0.67 \%$ yeast nitrogen base without amino acids, $20 \mathrm{mg} / \mathrm{L}$ uracil, $30 \mathrm{mg} / \mathrm{L}$ L-lysine $\mathrm{HCl}, 100 \mathrm{mg} / \mathrm{L}$ L-leucine, and $20 \mathrm{mg} / \mathrm{L} \mathrm{L}$-histidine $\mathrm{HCl}$ ) at $28^{\circ} \mathrm{C}$ with reciprocal shaking (120 rpm).

\section{Chemicals and Analysis Methods}

Exponentially growing yeast cells were harvested when $\mathrm{OD}_{600}$ of the culture medium reached 0.5 and were treated with various concentrations of ethanol (Wako, Osaka, Japan). Cycloheximide (CHX) was purchased from Nacalai Tesque, Kyoto, Japan. Polysome profile analysis was performed using a method described by Inada and Aiba (2005). The preparation of yeast extract and sucrose gradient separation for polysome profile analysis was performed using a gradient master and fractionator (107-201 M and 152-002, BioComp Instruments, Fredericton, $\mathrm{NB}$, Canada). Polysome ratio was determined as the percentage of area under polysomal ribosome peaks relative to that under total ribosome peaks, according to a method described by Hofmann et al. (2012). Fluorescence microscopic analysis was performed using a Leica AF6500 fluorescence microscope system (Leica Microsystems Vertrieb GmbH, Hessen, Germany).

\section{Plasmids}

Sequences of primers used for constructing plasmids are listed in Table 1. Genomic DNA from S. cerevisiae strain BY4742 was used as the template for amplifying yeast genes by PCR.

\section{YIp-BTN2-FLAG-BTN2 ${ }_{\mathrm{Ter}}$}

The integrate-type plasmid YIp-BTN2-FLAG was constructed to determine Btn2 protein expression. This plasmid contained a part of the BTN2 open reading frame (ORF), a FLAG tag sequence (encoded by $24 \mathrm{nt}$ ) immediately upstream of the stop codon, and a $3^{\prime}$-flanking region of BTN2. A $0.6-\mathrm{kbp}$ fragment encoding the part of $B T N 2$ Orf and a $0.2-\mathrm{kbp}$ fragment encoding $B T N 2$ FLAG-Ter were amplified using primer sets BTN2 $2_{\text {Orf }}-\mathrm{F} /$ BTN2 ${ }_{\text {Orf }}$ - R and BTN2 FLAG-Ter-F/BTN2 $_{\text {FLAG-Ter-R, }}$, respectively. Amplicons obtained were digested using XbaI/XhoI and $\mathrm{XhoI} / K p n \mathrm{I}$, respectively, and were cloned into the $\mathrm{XbaI} / \mathrm{Kp} n \mathrm{I}$ sites of pJK67 (Kahana et al., 1998) to construct YIp-BTN2FLAG-BTN2 ${ }_{\mathrm{Ter}}$. To integrate the BTN2-FLAG-BTN2 ${ }_{\mathrm{Ter}}$ gene in the chromosomal BTN2 locus, YIp-BTN2-FLAG-BTN2Ter was linearized by digesting it with $\mathrm{HpaI}$ and was introduced into yeast cells.

\section{YIp-BTN2-FLAG-ADH6 ${ }_{\mathrm{Ter}}$ and YIp-BTN2-FLAG-TEF1 ${ }_{\text {Ter }}$}

A 0.4 -kbp fragment encoding $A D H 6_{\text {FLAG-Ter }}$ and a $0.7-\mathrm{kbp}$ fragment encoding $T E F 1_{\text {FLAG-Ter }}$ were amplified using primer 
TABLE 1 | List of primers used in plasmid construction.

\begin{tabular}{|c|c|}
\hline Name & Sequence \\
\hline BTN2Orf-F & 5'-ACTITCTAGATTGGTITAGTTAAGCATGA-3' \\
\hline BTN2 Orf $-\mathrm{R}$ & 5'-TATCTCTCGAGATATCTCCTCAATAATAGA-3' \\
\hline BTN2 $2_{\text {FLAG-Ter }}-\mathrm{F}$ & 5'-GATATCTCGAGTCGACTACAAGGATGACGATGACAAGTAATGGGTGATAATACATACTCCCCATC-3' \\
\hline BTN2 FLAG-Ter-R & 5'-ATAATGGTACCAAAATCACGGATACTAATA-3' \\
\hline BTN2Pro 2-F & 5'-GGGGTACTAGTGCCTATAAGTTCGAAGCCA-3' \\
\hline BTN2Pro $2-R$ & 5'-GAAAAGAATTCTATATTGTAATGGGGTCTA-3' \\
\hline CUR1-F & 5'-AACCAAAAGAAAATAACTAATAGACCCCATTACAATATAGAAATGGCTGCCGCATGCATITGTCAACCTAATCTT-3' \\
\hline CUR1-R & 5'-TTATCACCCATTACTTGTCATCGTCATCCTTGTAGTCCTCGAGCCGCCCATTCAATCTTCTAGATACTTCCTT-3' \\
\hline GIC2-F & 5'-AACCAAAAGAAAATAACTAATAGACCCCATTACAATATAGAAATGACTAGTGCAAGTATTACCAATACTGGAAAC-3' \\
\hline GIC2-R & 5'-TTATCACCCATTACTTGTCATCGTCATCCTTGTAGTCCTCGAGAGTTTGCAGGGGCTCGAGCTGGTTGAAAGA-3' \\
\hline YUR1-F & 5'-AACC AAAAGAAAATAACTAATAGACCCCATTACAATATAGAAATGGCAAAAGGAGGCTCGCTATACATCGTTGGC-3’ \\
\hline YUR1-R & 5'-TTATCACCCATTACTTGTCATCGTCATCCTTGTAGTCCTCGAGAATCTCGTCTTGCTCTTCПITAAGAAATATTGCCG-3' \\
\hline ADH6FLAG-Ter-F & 5'-GCAAGCTCGAGCTGACTACAAGGATGACGATGACAAGTAGGTTGTCAAGCTCTTGATAAATG-3' \\
\hline ADH6 $F L A G-T e r-R$ & 5'-GAAAAGGTACCCAGATCTACCACCAAACCT-3' \\
\hline TEF1 ${ }_{\text {FLAG-Ter }}-\mathrm{F}$ & 5'-GCAAGCTCGAGCTGACTACAAGGATGACGATGACAAGTAAGGAGATTGATAAGACTITCTAG-3' \\
\hline TEF1 FLAG-Ter-R & 5'-CGTAAAACTAGATAGCAGTITGGTACCTAT-3' \\
\hline
\end{tabular}

sets $A D H 6_{\mathrm{FLAG}-\mathrm{Ter}}-\mathrm{F} / A D H 6_{\mathrm{FLAG}-\mathrm{Ter}} \mathrm{R}$ and $\mathrm{TEF} 1_{\mathrm{FLAG}-\mathrm{Ter}^{-}}$ $\mathrm{F} / \mathrm{TEF} 1_{\mathrm{FLAG}}$-Ter-R, respectively. Amplicons obtained were digested using XhoI/KpnI and were cloned into the XhoI/KpnI sites of YIp-BTN2-FLAG-BTN2 Ter to construct YIp-BTN2FLAG-ADH6 $6_{\mathrm{Ter}}$ and YIp-BTN2-FLAG-TEF1 ${ }_{\mathrm{Ter}}$, respectively. To integrate them in the chromosomal BTN2 locus, they were linearized with $\mathrm{HpaI}$ and introduced into yeast cells.

\section{pRS316-BTN2 Pro/FLAG-Ter}

A $1.2 \mathrm{kbp}$ fragment of the BTN2 promoter region (BTN2Pro) and a $0.2 \mathrm{kbp} B T N 2_{\mathrm{FLAG}}$-Ter fragment were amplified using primer sets BTN2 Pro $_{2-\mathrm{F} / B T N 2}$ Pro $_{2}$-R and BTN2 ${ }_{\mathrm{FLAG}-\mathrm{Ter}^{-}}$ $\mathrm{F} / B T N 2_{\mathrm{FLAG}-\mathrm{Ter}} \mathrm{R}$, respectively, and were cloned into the SpeI/EcoRI sites and the XhoI/KpnI sites, respectively, of pRS316 (Sikorski and Hieter, 1989) to construct pRS316BTN2Pro/FLAG-Ter, that was named pYY2712. ORFs of CUR1 (0.8 kbp), GIC2 (1.2 kbp), and YUR1 (1.3 kbp) were amplified using primer sets CUR1-F/CUR1-R, GIC2-F/GIC2-R, and YUR1-F/YUR1-R, respectively. Amplicons obtained were cloned into the pYY2712 that was previously digested with EcoRI/SalI to construct pYY plasmid series, pYY-CUR1-FLAG (pRS316-BTN2 Pro $-C U R 1_{\text {Orf- }}$ BTN2 ${ }_{\text {FLAG-Ter }}$ ), pYY-GIC2-FLAG (pRS316-BTN2 ${ }_{\text {Pro-GIC2 }}$ Orf-BTN2 ${ }_{\text {FLAG-Ter }}$ ), and pYY-YUR1FLAG (pRS316-BTN2Pro-YUR1 $1_{\text {Orf }}$-BTN2FLAG-Ter), respectively, by using gap repair cloning method (Goto and Nagano, 2013).

\section{Quantitative Reverse Transcription-PCR}

Relative mRNA levels of the BTN2, HSP30, CUR1-FLAG, GIC2FLAG, and YUR1-FLAG genes were determined by performing quantitative reverse transcription-PCR (qRT-PCR). Total RNA was extracted from yeast cells by using a method described by Schmitt et al. (1990). RNA obtained was reverse transcribed to cDNA by using ReverTra Ace qPCR RT Master Mix FSQ201 (Toyobo, Osaka, Japan), according to the manufacturer's instructions. Quantitative PCR was performed using Thermal
Cycler Dice Real Time System Lite (Takara Bio Inc., Shiga, Japan) and SYBR ${ }^{\circledR}$ Premix Ex Taq ${ }^{\mathrm{TM}}$ II (Takara Bio Inc., Shiga, Japan). Comparison of mRNA expression levels was performed by normalizing the mRNA level of each gene to that of ACT1 (Takahashi et al., 2011). The mRNA level was expressed as the ratio of normalized mRNA level of the target gene to that of the reference gene. Oligonucleotide sequences of primers used in qRT-PCR are listed in Table 2.

\section{Western Blotting}

After the treatment of cells with ethanol stress, cell-free extract (CFE) in $50 \mathrm{mM}$ potassium phosphate buffer ( $\mathrm{pH}$ 6.8) was prepared. Total protein concentration of the CFE was measured using Protein Assay CBB Solution kit (Nacalai Tesque, Kyoto, Japan). Next, the CFE was mixed with SDS sample buffer solution containing a reducing reagent $(6 \mathrm{x}$, Code No. 0949914; Nacalai Tesque) and was heated at $98^{\circ} \mathrm{C}$ for $5 \mathrm{~min}$. Thirty six micrograms of total protein was applied to each lane of a $10 \%$ polyacrylamide gel for the SDS-PAGE analysis. The resolved proteins were transferred onto polyvinylidene difluoride membranes (Immobilon-P; Merck Millipore Ltd,

\section{TABLE 2 | List of primers used in qRT-PCR.}

\begin{tabular}{ll}
\hline Name & Sequence \\
\hline ACT1-F & 5'-TTGGATTCCGGTGATGGTGTTACT-3' \\
ACT1-R & 5'-TGAAGAAGATTGAGCAGCGGTTG-3' \\
BTN2-F & 5'-TTCCGAAGGTGGCATC AAC-3' \\
BTN2-R & 5'-CTTCGCTTCTCCGCTTCTTC-3' \\
HSP30-F & $5^{\prime}$-TGGCCTGGATATGCAC ATTA-3' \\
HSP30-R & $5^{\prime}$-GACTGCAAACACTGCCCATA-3' \\
CUR1-F & 5'-CCTTCAATGGCAATGGCTTACA-3' \\
GIC2-F & 5'-GCTACGCCTTCTCCACAATCTA-3' \\
YUR1-F & 5'-ACCTGTCCAGCATCTTACGC-3' \\
FLAG-R & $5^{\prime}$-TC ATCGTCATCCTTGTAGTC-3'
\end{tabular}


MA, USA). The blotted membranes were blocked with PBS containing $0.05 \%$ Tween 20 and $1 \%$ skim milk for $10 \mathrm{~min}$. After washing, the membranes were incubated with anti-FLAG M2 primary antibody (dilution, 1:1,000; Sigma-Aldrich, MO, USA) to monitor the levels of FLAG-tagged proteins (Btn2, Cur1, Gic2, and Yur1). Pgk1 was used as a loading control, and its level was monitored using a monoclonal anti-Pgk1 primary antibody (22C5D8; dilution, 1:4,000; Life Technologies, Frederick, MD, USA). The anti-FLAG and anti-Pgk1 primary antibodies were detected using HRP-conjugated anti-mouse secondary antibody (dilution, 1:1,000; Cell Signaling Technology, Beverly, MA, USA). Antibody binding was detected using ChemiLumi One L Western blotting detection reagents (Code No. 07880-70; Nacalai Tesque). The bands of the Western blot were quantified using Image Studio Digits Ver 4.0 software (LICOR Biotechnology, Lincoln, NE, USA). Levels of FLAG-tagged proteins were normalized to that of Pgk1, and the intensity of the Pgk1 band in each lane was set at $100 \%$.
A
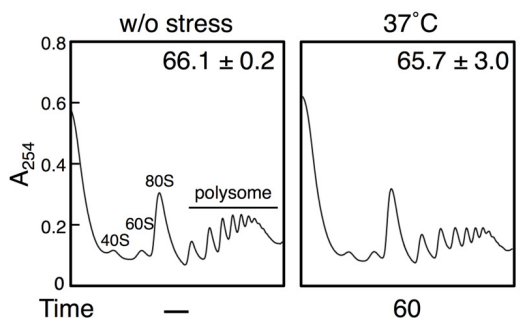

$5 \%$ Ethanol
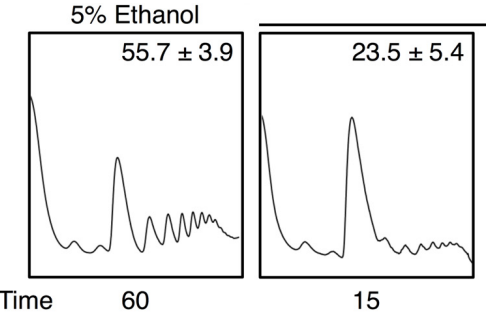

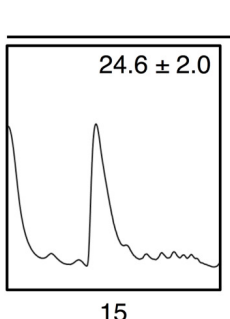

15

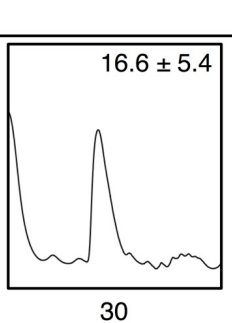

C

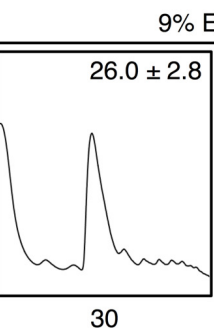

$10 \%$ Ethanol
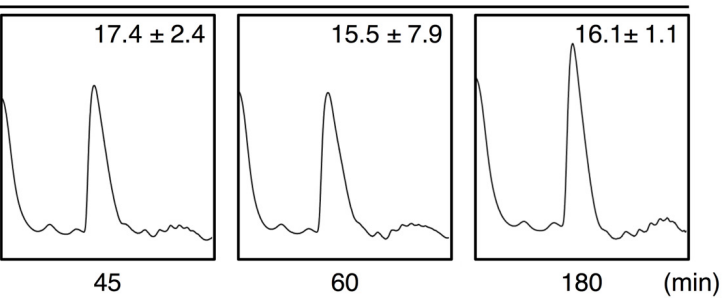

B
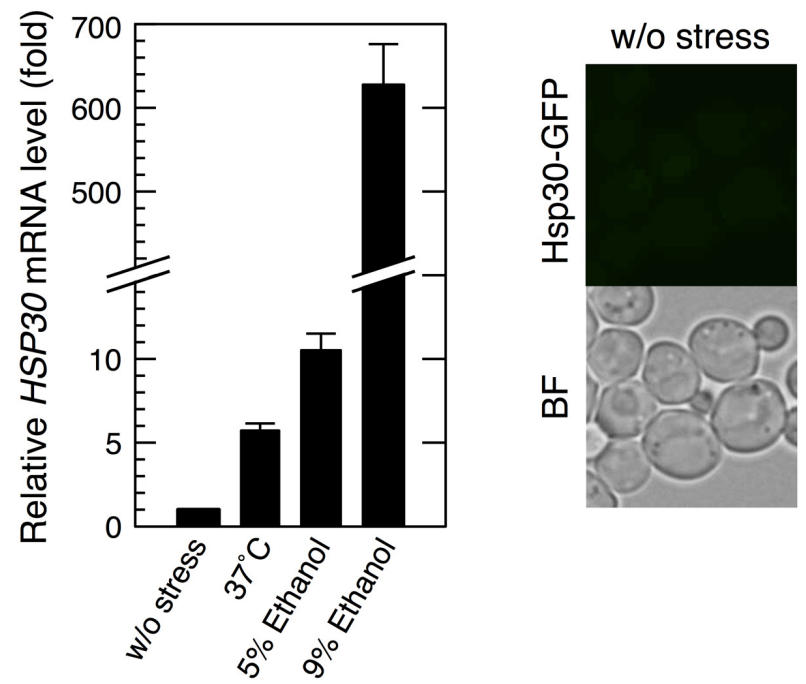

$37^{\circ} \mathrm{C}$

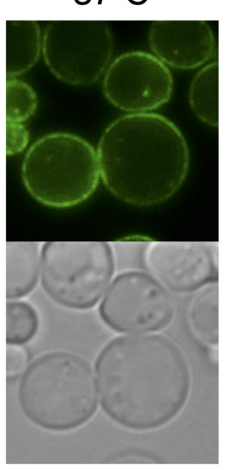

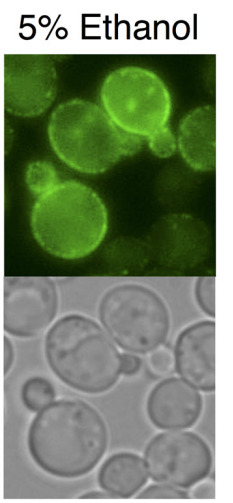

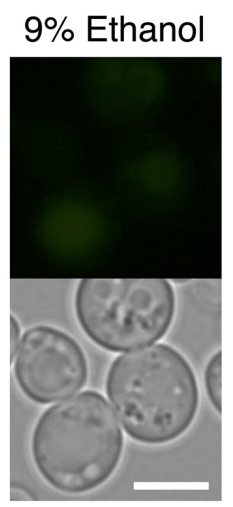

FIGURE 1 | Severe ethanol stress inhibited translation initiation in yeast cells. (A) The polysome profile of yeast cells under severe ethanol stress was determined. Yeast cells in the exponential growth phase in the SD medium at $28^{\circ} \mathrm{C}$ were treated with mild heat shock at $37^{\circ} \mathrm{C}$ for 60 min, $5 \%$ ethanol for $60 \mathrm{~min}$, or 9 or $10 \%$ (v/v) ethanol (severe ethanol stress) for the indicated time. Polysome, $40 \mathrm{~S}$ (small ribosomal subunit), $60 \mathrm{~S}$ (large ribosomal subunit), and $80 \mathrm{~S}$ (monosome) peaks are labeled. The numbers in the panels indicate the percentages of polysomal ribosomes. Data are expressed as mean $\pm \mathrm{SD}(n=3)$. (B,C) Yeast cells carrying the GFP-tagged chromosomal copy of $H S P 30$ were treated with 5 or $9 \%$ ethanol stress or with mild heat shock at $37^{\circ} \mathrm{C}$ for 60 min. (B) HSP30 mRNA level was analyzed by performing qRT-PCR and was normalized with that of ACT1. Each value is expressed as the mean \pm SD of relative fold change in expression normalized to that in untreated control cells $(n=3)$. (C) Hsp30-GFP synthesis was detected by performing fluorescence microscopic analysis. Cells under stress were observed immediately after treatment without fixation. Exposure time is the same in all the images. The white bar indicates $5 \mu \mathrm{m}$. BF, bright field. 


\section{RESULTS}

\section{High Concentration Ethanol Stress Caused the Repression of Translation Activity}

First, we performed polysome profile analysis to verify whether severe ethanol stress repressed translation activity in yeast cells (Figure 1A). Treatment of yeast cells with 9 or $10 \%(\mathrm{v} / \mathrm{v})$ ethanol induced a pronounced rapid reduction in polysome fraction and a concomitant increase in monosome fraction (80S), indicating a significant repression of translation initiation, which was similar to that observed during glucose deprivation (Ashe et al., 2000). Further, prolonged treatment of yeast cells with $10 \%$ ethanol maintained ethanol stress-induced translation repression for at least $180 \mathrm{~min}$. These data clearly confirmed that severe ethanol stress ( $>9 \%$ ethanol, v/v) considerably repressed overall protein synthesis in yeast cells. Although mRNA levels of HSP30 increased in yeast cells treated with $9 \%$ ethanol for $60 \mathrm{~min}$ (Figure 1B), Hsp30-GFP protein expression was negligible as indicated by very weak fluorescence intensity
(Figure 1C). However, exposure to heat shock at $37^{\circ} \mathrm{C}$ or treatment with 5\% ethanol (mild ethanol stress) induced Hsp30GFP protein expression in the plasma membrane of yeast cells (Figure 1C), indicating that severe ethanol stress repressed Hsp30 protein synthesis. These results confirmed that increased mRNA expression did not necessarily indicate an increase in corresponding protein expression in yeast cells under severe ethanol stress because of the repression of overall protein synthesis.

\section{BTN2 Was Preferentially Translated under Severe Ethanol Stress}

We next tried to identify genes that were preferentially translated under severe ethanol stress. Since various genes associated with ethanol tolerance have been reported (Alexandre et al., 2001; Takahashi et al., 2001; Fujita et al., 2006; van Voorst et al., 2006; Hirasawa et al., 2007; Teixeira et al., 2009), we randomly examined whether protein levels of those genes were increased by the treatment with 9 or $10 \%$ ethanol. As a screening result, only BTN2 was confirmed as the gene whose protein levels
A

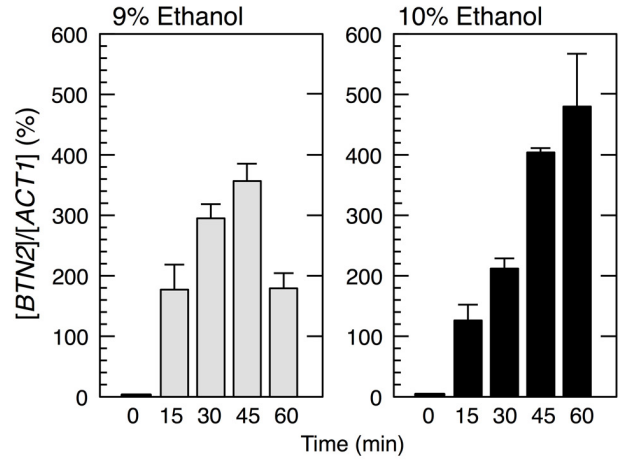

B

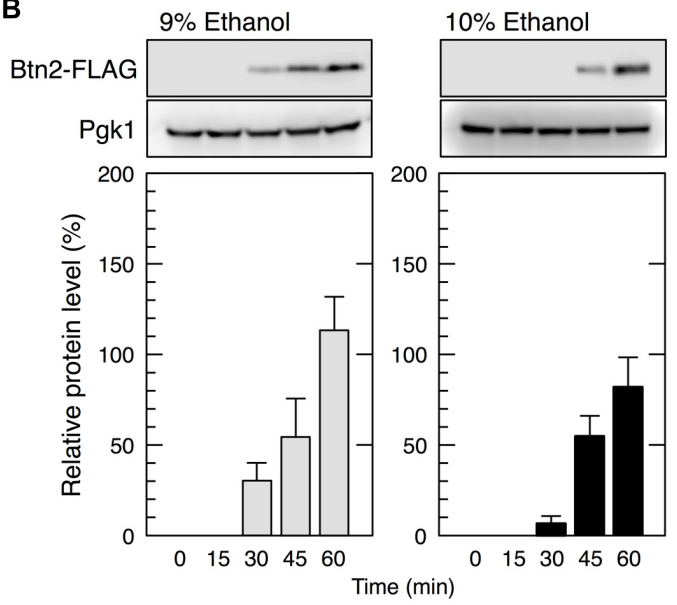

C

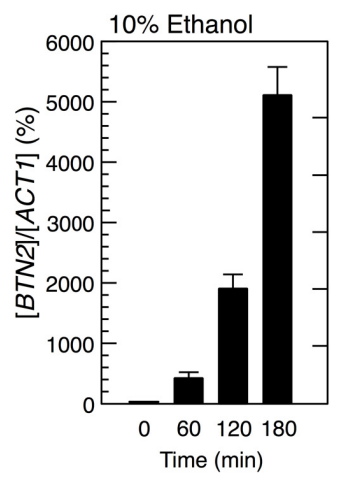

D

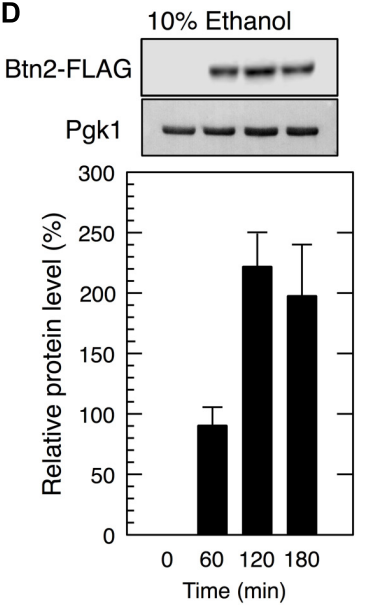

E

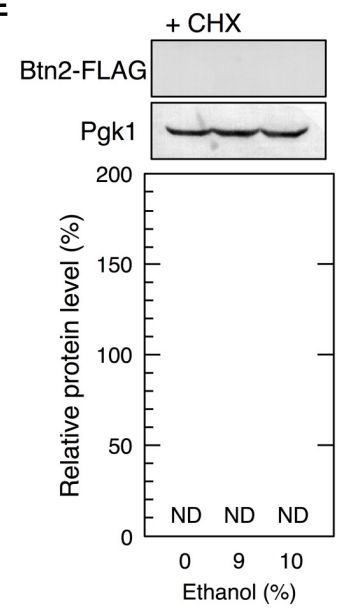

FIGURE 2 | BTN2 was preferentially expressed under severe ethanol stress. Yeast cells carrying a FLAG-tagged chromosomal copy of BTN2 in the exponential growth phase were treated with 9 or $10 \%(\mathrm{~V} / \mathrm{V})$ ethanol (severe ethanol stress) for the indicated time (A-D) or treated with 9 or $10 \%(\mathrm{~V} / \mathrm{V})$ ethanol and $0.1 \mathrm{mg} / \mathrm{ml}$ cycloheximide (CHX) for $60 \mathrm{~min}$ (E). (A,C) BTN2 mRNA level was determined by performing qRT-PCR and was normalized to that of ACT1. (B,D,E) Btn2 protein level was determined by performing Western blotting with anti-FLAG antibody. Pgk1 was used as the loading control. Btn2 protein level was normalized to that of Pgk1, and the intensity of the Pgk1 band in each lane was set at 100\%. Data are expressed as mean \pm SD $(n=3)$. ND, not detected. 


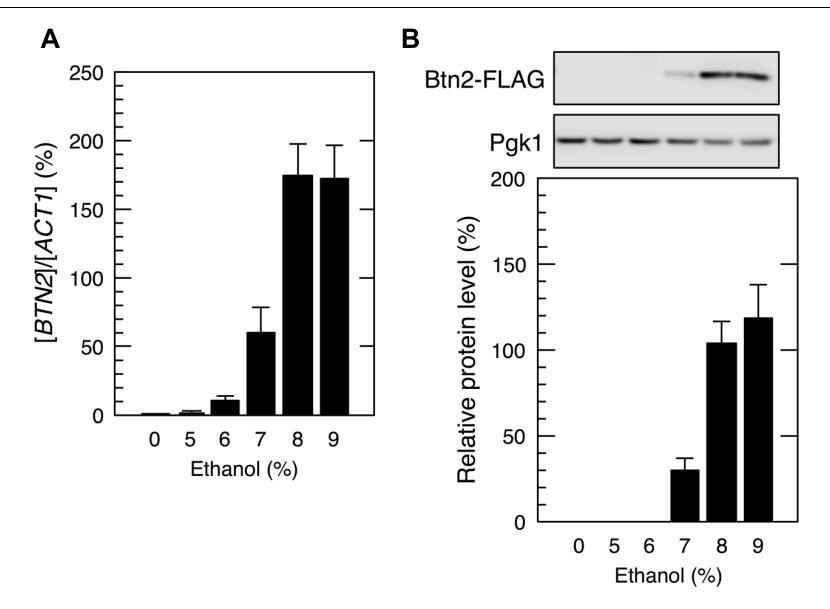

FIGURE 3 | BTN2 expression under mild ethanol stress. Cells carrying a FLAG-tagged chromosomal copy of BTN2 gene in the exponential growth phase were treated with the indicated concentrations of ethanol for $60 \mathrm{~min}$. (A) BTN2 mRNA level was determined by performing qRT-PCR and was normalized to that of ACT1. (B) Btn2 protein level was determined by performing Western blotting with anti-FLAG antibody. Pgk1 was used as the loading control. Btn2 protein level was normalized to that of Pgk1, and the intensity of the Pgk1 band in each lane was set at 100\%. Data are expressed as mean $\pm \operatorname{SD}(n=3)$. were significantly increased under severe ethanol stress so far. BTN2 encodes a v-SNARE binding protein and is associated with ethanol tolerance (Chattopadhyay and Pearce, 2002; Kama et al., 2007; Espinazo-Romeu et al., 2008; Yang et al., 2011). We first examined BTN2 mRNA level in yeast cells under severe ethanol stress ( 9 or $10 \%$ ethanol). Our results showed that BTN2 mRNA level significantly increased under severe ethanol stress (Figure 2A), which was similar to that reported by Cho et al. (2014).

We next examined whether Btn2 protein synthesis was upregulated under severe ethanol stress (Figure 2B). Although Btn2 protein expression was negligible in yeast cells under nonstress condition, clear bands representing Btn2 were detected following the treatment with severe ethanol stress. Btn2 protein levels significantly increased within $30 \mathrm{~min}$ in cells treated with $9 \%$ ethanol and within 45 min in cells treated with $10 \%$ ethanol. Prolonged treatment with $10 \%$ ethanol further increased Btn2 protein levels (Figures 2C,D). These results strongly suggested that BTN2 expression was induced and that BTN2 mRNA was efficiently translated in yeast cells under severe ethanol stress, despite the pronounced repression of overall protein synthesis. To verify translation of BTN2 mRNA under severe ethanol stress, we also investigated protein levels of Btn2 in cells simultaneously treated with severe ethanol
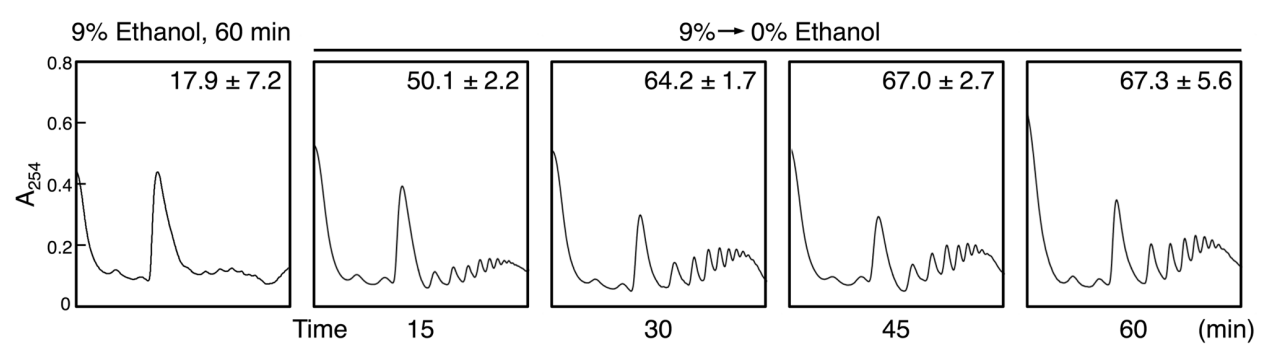

$10 \%$ Ethanol, $60 \mathrm{~min}$
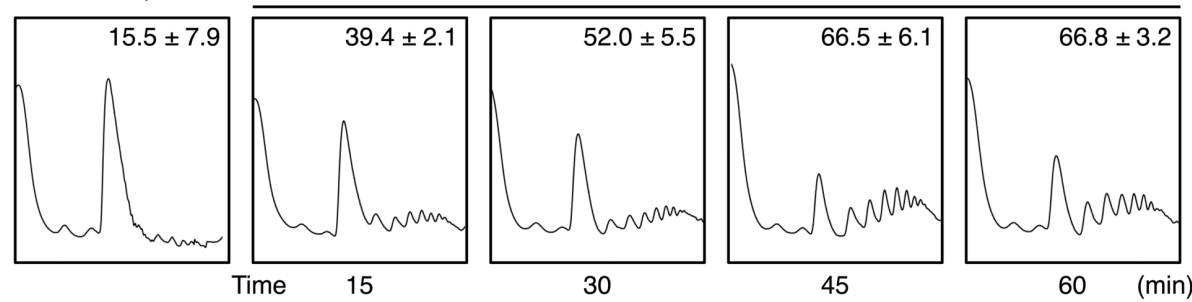

$10 \% \rightarrow 5 \%$ Ethanol
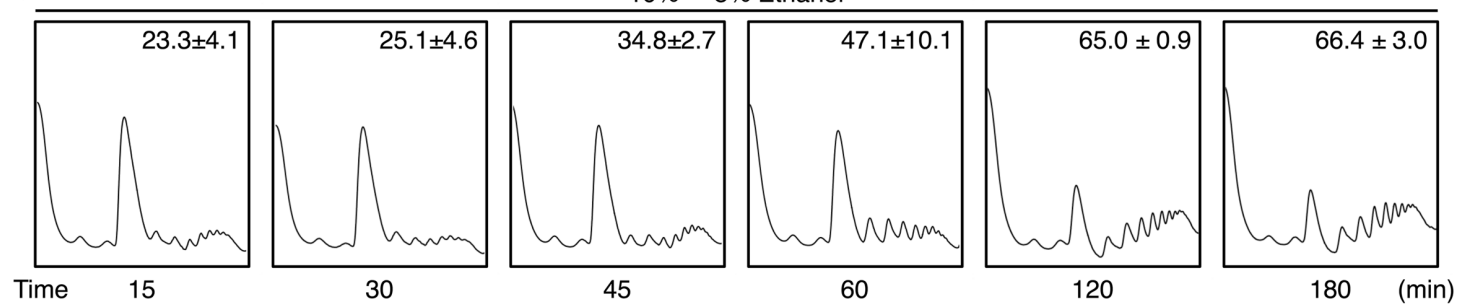

FIGURE 4 | Reconstruction of polysomes during recovery from severe ethanol stress. Yeast cells in the exponential growth phase were treated with 9 or $10 \%$ (v/v) ethanol (severe ethanol stress) for 60 min. After the treatment with ethanol stress, cells were transferred to a fresh SD medium lacking ethanol or containing $5 \%$ ethanol and were incubated at $28^{\circ} \mathrm{C}$ for the indicated time. The numbers in the panels indicate the percentages of polysomal ribosomes. Data are expressed as mean $\pm \operatorname{SD}(n=3)$. 
stress and cycloheximide (CHX), a strong inhibitor of protein biosynthesis (Obrig et al., 1971). CHX had almost no effect on the transcriptional activation of BTN2 (data not shown) but completely repressed the increase of Btn2 protein levels (Figure 2E) under severe ethanol stress. These results clearly indicate that newly synthesis of Btn2 protein was induced under severe ethanol stress.

In contrast, yeast cells exposed to mild ethanol stress (5 and $6 \%$ ethanol, v/v) for 60 min did not show the efficient translation of BTN2 mRNA (Figure 3), indicating that BTN2 was expressed under severe ethanol stress but not under mild ethanol stress.

\section{Elimination of Ethanol Stress Reconstructed Polysomes and Inhibited Btn2 Protein Expression}

We next investigated the restoration of translation activity during recovery from severe ethanol stress (Figure 4). Elimination of ethanol by replacing the culture medium (decrease in ethanol concentration from 9 or 10 to $0 \%$ ) significantly reduced the monosome (80S) fraction and markedly increased the polysome fraction, indicating the restoration of translation activity. Medium replacement efficiently increased the percentage of polysomal ribosomes to the original level within 30$45 \mathrm{~min}$. These results suggested that severe ethanol stressinduced translation repression was smoothly recovered after the elimination of ethanol stress. Next, we examined Btn2 protein levels in yeast cells during the recovery process from severe ethanol stress. Medium replacement not only decreased BTN2 mRNA levels but also gradually decreased Btn2 protein levels, with negligible Btn 2 protein levels being detected within $45 \mathrm{~min}$ after medium replacement (Figures 5A,B).

However, culturing of $10 \%$ ethanol-treated yeast cells in a fresh medium containing 5\% ethanol (from 10 to $5 \%$ ethanol) did not completely restore the translation activity within $1 \mathrm{~h}$ (Figure 4). The time required for the reconstruction of polysomes and inhibition of Btn2 protein expression was longer in yeast cells cultured in SD medium containing 5\% ethanol than in yeast cells cultured in SD medium lacking ethanol (Figure 5).

\section{The BTN2 Promoter Region Induces Protein Synthesis under Severe Ethanol Stress}

Promoter regions of some genes induce the preferential translation of non-native genes during severe stress-induced translation repression. Zid and O'Shea (2014) reported that the promoters of HSP26 and HSP30 increased protein synthesis during glucose deprivation. We also recently reported that the $A D H 7$ promoter and $B D H 2$ promoter induced translation under severe vanillin stress, despite the pronounced repression of overall protein synthesis (Nguyen et al., 2015; Ishida et al., 2016). Based on these findings, we investigated whether the BTN2 promoter induced the protein synthesis of non-native genes under severe ethanol stress.

We constructed an expression system containing the promoter and terminator regions of BTN2 (pYY2712, see
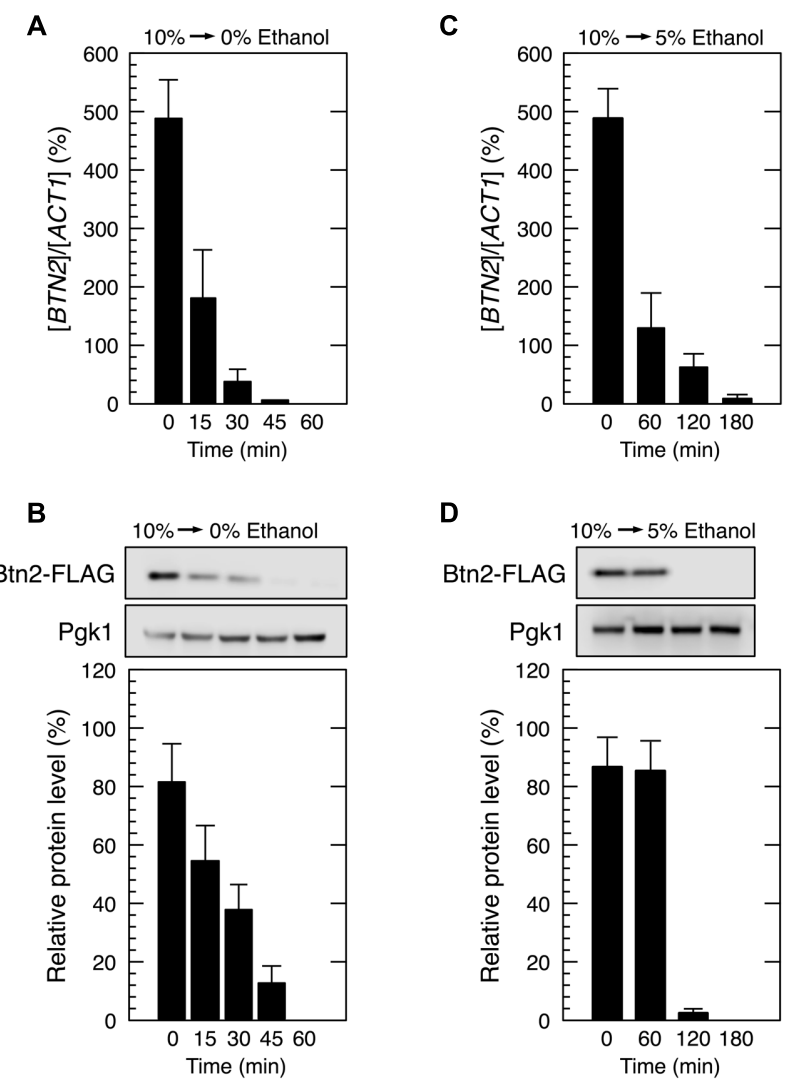

FIGURE 5 | Btn2 protein level decreased during recovery from severe ethanol stress. Cells carrying a FLAG-tagged chromosomal copy of BTN2 gene in the exponential growth phase were treated with severe ethanol stress $(10 \% \mathrm{v} / \mathrm{v})$ for $60 \mathrm{~min}$. After the treatment with ethanol stress, cells were transferred to a fresh SD medium lacking ethanol $(\mathbf{A}, \mathbf{B})$ or containing $5 \%$ ethanol (C,D) and were incubated further at $28^{\circ} \mathrm{C}$ for the indicated time. $(\mathbf{A}, \mathbf{C})$ BTN2 mRNA level was determined by performing $\mathrm{QRT}$-PCR and was normalized to that of ACT1. (B,D) Btn2 protein level was determined by performing Western blotting with anti-FLAG antibody. Pgk1 was used as the loading control. Btn2 protein level was normalized to that of Pgk1, and the intensity of the Pgk1 band in each lane was set at 100\%. Data are expressed as mean $\pm \operatorname{SD}(n=3)$.

Materials and Methods) flanking the ORFs of target genes. We examined three genes, CUR1, GIC2, and YUR1. CUR1 was chosen as a paralog of BTN2 (Byrne and Wolfe, 2005; Malinovska et al., 2012). GIC2 and YUR1 were randomly chosen from yeast genes. Yeast cells harboring pYY-CUR1-FLAG showed increased protein levels of Cur1, a sorting factor and central regulator of spatial protein quality control (Kryndushkin et al., 2008), under severe ethanol stress (Figure 6). Similarly, yeast cells carrying pYY-GIC2-FLAG or pYY-YUR1-FLAG showed increased protein levels of Gic2, a rho-like GTPase Cdc42 effector (Chen et al., 1997), or Yur1, a mannosyltransferase (Lussier et al., 1997), under severe ethanol stress. Since replacement of the $B T N 2$ terminator region with other terminator regions (ADH6 terminator region and TEF1 terminator region) did not affect the induction of Btn2 protein synthesis (Figure 6C), contribution of the BTN2 terminator to the preferential translation of BTN2 
A

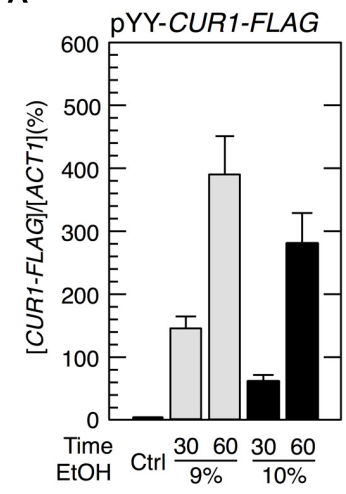

B
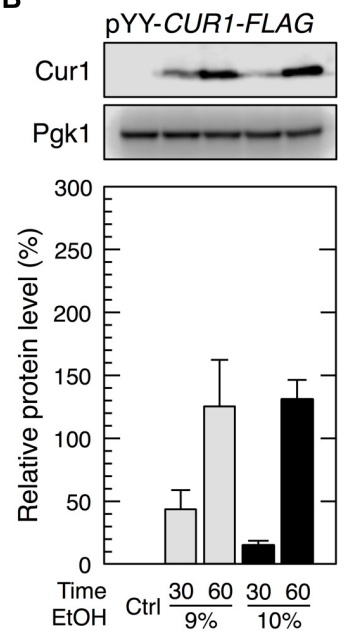
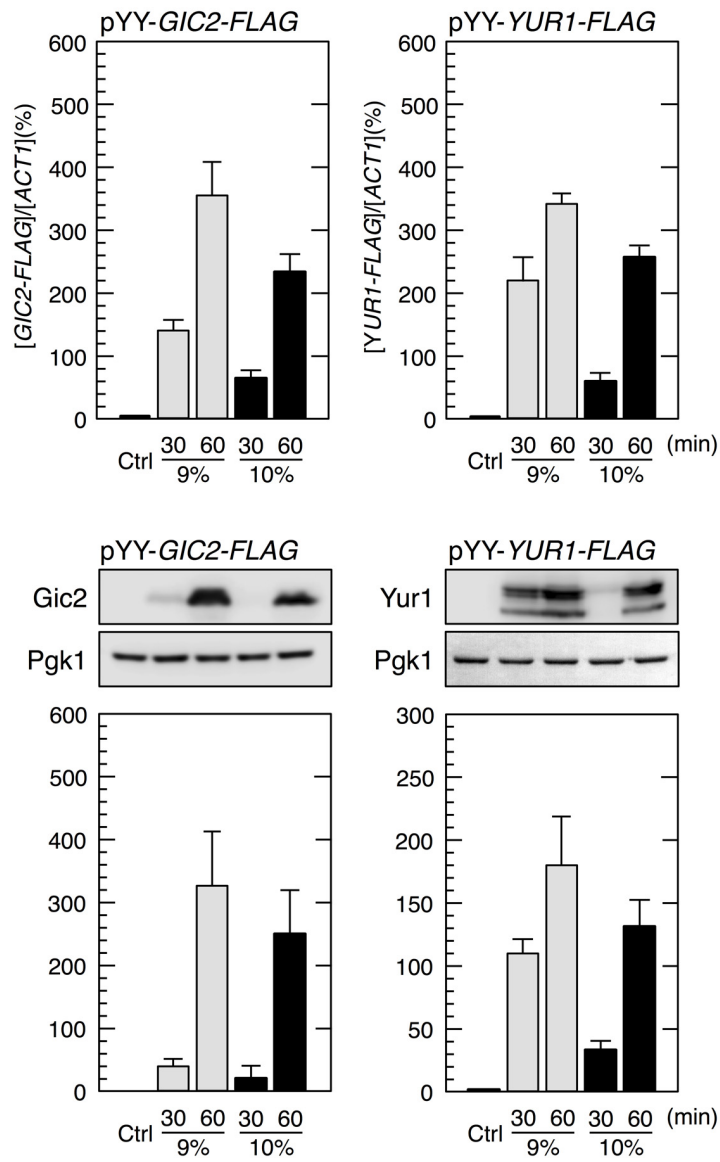
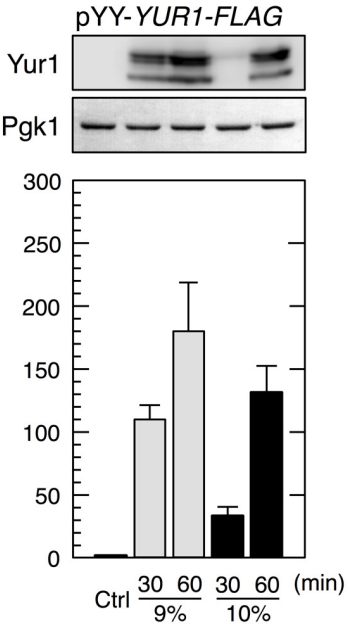

C
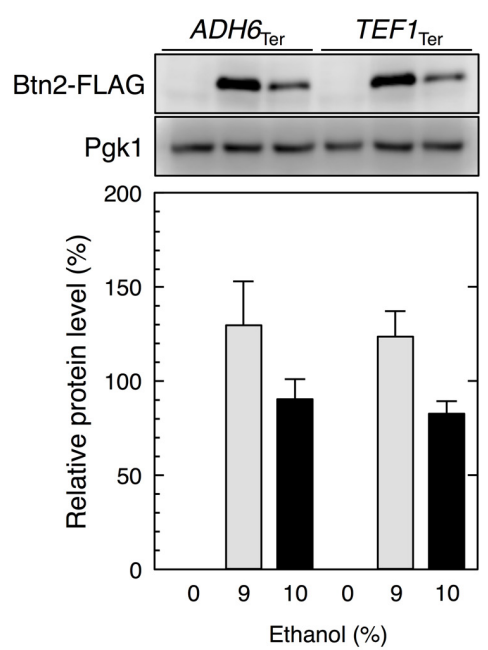

FIGURE 6 | The BTN2 promoter region induced the protein synthesis of non-native genes under severe ethanol stress. (A,B) Cells harboring the pYY plasmid series (BTN2 promoter-driven expression system) in the exponential growth phase were treated with 9 or $10 \%$ ethanol for the indicated time. (A) The mRNA levels of each FLAG-tagged gene were analyzed by performing qRT-PCR and were normalized to that of ACT1. (B) Protein levels of Cur1-FLAG, Gic2-FLAG, and Yur1-FLAG were determined by performing Western blotting with anti-FLAG antibody. (C) The BTN2 terminator region had a negligible effect on preferential translation upon severe ethanol stress. Cells carrying Ylp-BTN2-FLAG-ADH6 Ter or Ylp-BTN2-FLAG-TEF1 Ter in the exponential phase of growth were treated with 9 or $10 \%$ ethanol for $1 \mathrm{~h}$. Protein levels of Btn2-FLAG were determined by Western blot analysis using an anti-FLAG antibody. Pgk1 was used as the loading control. Their protein levels were normalized to that of Pgk1, and the intensity of the Pgk1 band in each lane was set at $100 \%$. Data are shown as mean \pm SD ( $n=3)$. Ctrl, control cells not treated with ethanol stress.

under severe ethanol stress seems negligible. These results strongly suggested that the BTN2 promoter region induced the expression of its regulated genes under severe ethanol stress.

\section{DISCUSSION}

In the present study, we performed polysome profile analysis to verify that high ethanol concentration severely repressed the bulk translation activity in yeast cells. The pronounced translation repression was maintained for at least $180 \mathrm{~min}$ after exposure to $10 \%$ ethanol stress (Figure 1A). Compared with high salinity stress $(1 \mathrm{M} \mathrm{NaCl})$, which induces pronounced but transient translation repression (Uesono and Toh-E, 2002; Melamed et al., 2008), severe ethanol stress induced long-term translation repression. However, elimination of ethanol stress rapidly restored translation activity (Figure 4), clearly indicating that severe ethanol stress did not irreparably damage the components of the translation apparatus.

Next, we tried to identify mRNAs that overcome severe ethanol stress-induced translation repression and found that BTN2 mRNA was preferentially translated despite the pronounced repression of overall protein synthesis (Figure 2). The null mutant of BTN2 is hypersensitive to ethanol (EspinazoRomeu et al., 2008; Yang et al., 2011), suggesting that BTN2 expression through its preferential translation is necessary for inducing resistance to severe ethanol stress. However, severe ethanol stress-induced Btn2 protein expression was abolished after eliminating ethanol stress (Figure 5). It has been reported that Btn2 protein levels were low and detection of Btn2 was relatively difficult under physiological growth conditions (Chattopadhyay and Pearce, 2002; Kryndushkin et al., 2008; Malinovska et al., 2012; Miller et al., 2015). Consistently, we also observed that Btn2 protein levels were very low under 
mild ethanol stress (Figure 3). Additionally, overexpression of BTN2 causes the delay of cell growth (Sopko et al., 2006). These previous reports and our results suggested that BTN2 is expressed under severe ethanol stress but not under mild ethanol stress and that BTN2 expression is strictly repressed under non-severe ethanol stress conditions.

Heat shock elements in the promoter regions are crucial for inducing preferential translation under glucose deprivation (Zid and O'Shea, 2014). The BTN2 promoter region contains three HSEs, namely, HSE1, 5' -AGAAAGTTCCGGAAA-3' (from -340 to -326 relative to the translation initiation ATG codon); HSE2, 5'-GGAAGAATTAAGAATTTCATAGAAG-3' (from -230 to -206); and HSE3, 5'-ATGGAAGA-3' (from -170 to -163) (Slater and Craig, 1987; Yamamoto et al., 2005; Badis et al., 2008). These HSEs are crucial for the transcriptional activation of BTN2 under severe ethanol stress (Cho et al., 2014). Additionally, they may play a role in the prioritized translation of BTN2 under severe ethanol stress as well as glucose deprivation. A recent study reported that mammalian translation elongation factor eEF1A is recruited to HSEs in the HSP70 promoter region to promote Hsp70 protein synthesis by coupling transcription with translation during heat shock-induced translation repression (Vera et al., 2014). Similarly, some factor(s) that promote efficient translation under severe stress might be recruited to HSEs in yeast cells. However, presence of HSEs in promoter regions may not be sufficient for inducing effective translation under severe ethanol stress, unlike that observed during glucose deprivation, because translation of HSE-containing genes such as HSP30 and HSP82 is negligible under severe ethanol stress despite of their transcription (Figure 1) (Izawa et al., 2008). Mechanisms underlying preferential translation under severe ethanol stress might be different from those underlying preferential translation under glucose deprivation. Identification of essential cis- and trans-elements for preferential translation under severe ethanol stress is in progress. Identification of the essential cis-element(s) in the BTN2 promoter regions will facilitate the identification of

\section{REFERENCES}

Alexandre, H., Ansanay-Galeote, V., Dequin, S., and Blondin, B. (2001). Global gene expression durig short-term ethanol stress in Saccharomyces cerevisiae. FEBS Lett. 498, 98-103. doi: 10.1016/S0014-5793(01)02503-0

Ashe, M. P., De Long, S. K., and Sachs, A. B. (2000). Glucose depletion rapidly inhibits translation initiation in yeast. Mol. Biol. Cell 11, 833-848. doi: 10.1091/mbc.11.3.833

Badis, G., Chan, E. T., van Bakel, H., Pena-Castillo, L., Tillo, D., Tsui, K., et al. (2008). A library of yeast transcription factor motifs reveals a widespread function for Rsc3 in targeting nucleosome exclusion at promoters. Mol. Cell 32, 878-887. doi: 10.1016/j.molcel.2008.11.020

Balagopal, V., and Parker, R. (2009). Polysomes, P bodies and stress granules: states and fates of eukaryotic mRNAs. Curr. Opin. Cell Biol. 21, 403-408. doi: 10.1016/j.ceb.2009.03.005

Buchan, J. R., and Parker, R. (2009). Eukaryotic stress granules: the ins and outs of translation. Mol. Cell 36, 932-940. doi: 10.1016/j.molcel.2009.11.020

Buchan, J. R., Yoon, J. H., and Parker, R. (2011). Stress-specific composition, assembly and kinetics of stress granules in Saccharomyces cerevisiae. J. Cell Sci. 124, 228-239. doi: 10.1242/jcs.078444

Byrne, K. P., and Wolfe, K. H. (2005). The yeast gene order browser: combining curated homology and syntenic context reveals gene fate in polyploid species. Genome Res. 15, 1456-1461. doi: 10.1101/gr.3672305 other genes that are preferentially translated under severe ethanol stress.

We successfully expressed genes other than BTN2 under severe ethanol stress by using the BTN2 promoter region (Figure 6). The ability of the BTN2 promoter to overcome translation repression induced by severe ethanol stress could be very useful for improving the fermentation ability of yeast cells. For example, the BTN2 promoter could be used to express genes important for ethanol production, which are usually repressed under ethanol stress, for increasing and stabilizing the fermentation activity of yeast cells. The BTN2 promoter could also be used to alter the quality of wine and sake by modifying gene expression pattern in the final stage of brewing. Furthermore, utilization of the BTN2 promoter might realize efficient parallel-production of ethanol and useful proteins such as vaccines and hormones during wine making and Japanese sake brewing.

\section{AUTHOR CONTRIBUTIONS}

YY did most of experiments and SI did several experiments and mainly prepared the manuscript.

\section{FUNDING}

The Japan Society for the Promotion of Science 26292039, $15 \mathrm{~K} 0737$, and 20260614 are for SI.

\section{ACKNOWLEDGMENTS}

We are grateful to S. Kato, A. Iwaki, and T. T. M. Nguyen for their technical assistance. We would like to thank Editage for English language editing.

Chattopadhyay, S., Muzaffar, N. E., Sherman, F., and Pearce, D. A. (2000). The yeast model for Batten disease: mutations in btn1, btn2, and hsp30 alter pH homeostasis. J. Bacteriol. 182, 6418-6423. doi: 10.1128/JB.182.22.64186423.2000

Chattopadhyay, S., and Pearce, D. A. (2002). Interaction with Btn2p is required for localization of Rsglp: Btn2p-mediated changes in arginine uptake in Saccharomyces cerevisiae. Eukaryot. Cell 1, 606-612. doi: 10.1128/EC.1.4.606612.2002

Chen, G. C., Kim, Y. J., and Chen, C. S. (1997). The Cdc42 GTPaseassociated proteins Gic1 and Gic2 are required for polarized cell growth in Saccharomyces cerevisiae. Genes Dev. 11, 2958-2971. doi: 10.1101/gad.11.22. 2958

Cho, B., Lee, P., and Hahn, J. (2014). CK2-dependent inhibitory phosphorylation is relieved by Pptl phosphatase for the ethanol stress-specific activation of Hsf1 in Saccharomyces cevervisiae. Mol. Microbiol. 93, 306-316. doi: $10.1111 / \mathrm{mmi} .12660$

Espinazo-Romeu, M., Cantoral, J. M., Matallana, E., and Aranda, A. (2008). Btn2p is involved in ethanol tolerance and biofilm formation in flor yeast. FEMS Yeast Res. 8, 1127-1136. doi: 10.1111/j.1567-1364.2008.00397.x

Fujita, K., Matsuyama, A., Kobayashi, Y., and Iwahashi, H. (2006). The genomewide screening of yeast deletion mutants to identify the genes required for tolerance to ethanol and other alcohols. FEMS Yeast Res. 6, 744-750. doi: 10.1111/j.1567-1364.2006.00040.x 
Goto, K., and Nagano, Y. (2013). Ultra-low background DNA cloning system. PLoS ONE 8:e56530. doi: 10.1371/journal.pone.0056530

Grousl, T., Ivanov, P., Frydlova, I., Vasicova, P., Janda, F., Vojtova, J., et al. (2009). Robust heat shock induces eIF2-phosphorylation-independent assembly of stress granules containing eIF3 and $40 \mathrm{~S}$ ribosomal subunits in budding yeast, Saccharomyces cerevisiae. J. Cell Sci. 122, 2078-2088. doi: 10.1242/jcs. 045104

Hirasawa, T., Yoshikawa, K., Nakakura, Y., Nagahisa, K., Furusawa, C., Katakura, Y., et al. (2007). Identification of target genes conferring ethanol stress tolerance to Saccharomyces cerevisiae based on DNA microarray data analysis. J. Biotechnol. 131, 34-44. doi: 10.1016/j.jbiotec.2007.05.010

Hofmann, S., Cherkasova, V., Bankhead, P., Bukau, B., and Stoecklin, G. (2012). Translation suppression promotes stress granule formation and cell survival in response to cold shock. Mol. Biol. Cell 23, 3786-3800. doi: 10.1091/mbc.E12-040296

Inada, T., and Aiba, H. (2005). Translation of aberrant mRNAs lacking a termination codon or with a shortened $3^{\prime}$-UTR is repressed after initiation in yeast. $E M B O ~ J .24,1584-1595$. doi: $10.1038 /$ sj.emboj.7600636

Ishida, Y., Nguyen, T. T. M., Kitajima, S., and Izawa, S. (2016). Prioritized expression of $B D H 2$ under bulk translational repression and its contribution to tolerance to severe vanillin stress in Saccharomyces cerevisiae. Front. Microbiol. 7:1059. doi: 10.3389/fmicb.2016.01059

Iwaki, A., Kawai, T., Yamamoto, Y., and Izawa, S. (2013). Biomass conversion inhibitors, furfural and 5-hydroxymethylfurfural, induce the formation of mRNP granules and attenuate translation activity in yeast. Appl. Environ. Microbiol. 446, 225-233. doi: 10.1128/AEM.02797-12

Izawa, S. (2010). Ethanol stress response in mRNA flux of Saccharomyces cerevisiae. Biosci. Biotechnol. Biochem. 74, 7-12. doi: 10.1271/bbb.90686

Izawa, S. (2015). "Yeast mRNA flux during brewing and under ethanol stress conditions," in Stress Biology of Yeast and Fungi, eds H. Takagi and H. Kitagaki (Tokyo: Springer), 43-57.

Izawa, S., Kita, T., Ikeda, K., and Inoue, Y. (2008). Heat shock and ethanol stress provoke distinctly different responses in $3^{\prime}$-processing and nuclear export of HSP mRNA in Saccharomyces cerevisiae. Biochem. J. 414, 111-119. doi: 10.1042/BJ20071567

Izawa, S., Kita, T., Ikeda, K., Miki, T., and Inoue, Y. (2007). Formation of the cytoplasmic P-bodies in sake yeast during Japanese sake brewing and wine making. Biosci. Biotechnol. Biochem. 71, 2800-2807. doi: 10.1271/bbb. 70417

Izawa, S., Takemura, R., Ikeda, K., Fukuda, K., Wakai, Y., and Inoue, Y. (2005a). Characterization of Rat8 localization and mRNA export in Saccharomyces cerevisiae during the brewing of Japanese sake. Appl. Microbiol. Biotechnol. 69, 86-91. doi: 10.1007/s00253-005-1954-x

Izawa, S., Takemura, R., Miki, T., and Inoue, Y. (2005b). Characterization of the export of bulk poly(A) ${ }^{+}$mRNA in Saccharomyces cerevisiae during the wine-making process. Appl. Environ. Microbiol. 71, 2179-2182. doi: 10.1128/AEM.71.4.2179-2182.2005

Kahana, J. A., Schlenstedt, G., Evanchuk, D. M., Geiser, J. R., Hoyt, M. A., and Silver, P. A. (1998). The yeast dynactin complex is involved in partitioning the mitotic spindle between mother and daughter cells during anaphase B. Mol. Biol. Cell 9, 1741-1756. doi: 10.1091/mbc.9.7.1741

Kama, R., Robinson, M., and Gerst, J. E. (2007). Btn2, a hook1 ortholog and potential batten disease-related protein, mediates late endosome-Golgi protein sorting in yeast. Mol. Cell. Biol. 27, 605-621. doi: 10.1128/MCB.00699-06

Kato, K., Yamamoto, Y., and Izawa, S. (2011). Severe ethanol stress induces assembly of stress granules in Saccharomyces cerevisiae. Yeast 28, 339-347. doi: 10.1002/yea. 1842

Kim, Y., Chattopadhyay, S., Locke, S., and Pearce, D. A. (2005). Interaction among $\operatorname{Btn} 1 p, B \operatorname{tn} 2 p$, and Ist2p reveals potential interplay among the vacuole, amino acid levels, and ion homeostasis in the yeast Saccharomyces cerevisiae. Eukaryot. Cell 4, 281-288. doi: 10.1128/EC.4.2.281-288.2005

Kryndushkin, D. S., Shewmaker, F., and Wickner, R. B. (2008). Curing of the [URE3] prion by Btn2p, a Batten disease-related protein. EMBO J. 27, 27252735. doi: 10.1038/emboj.2008.198

Lussier, M., Sdicu, A. M., Camirand, A., and Bussey, H. (1997). Functional characterization of the YUR1, KTR1, and KTR2 genes as members of the yeast KRE2/MNT1 mannosyltransferase gene family. J. Biol. Chem. 271, 1100111008. doi: $10.1074 /$ jbc. 271.18 .11001
Malinovska, L., Kroschwald, S., Munder, M. C., Richter, D., and Alberti, S. (2012). Molecular chaperones and stress-inducible protein-sorting factors coordinate the spatiotemporal distribution of protein aggregates. Mol. Biol. Cell 23, 30413056. doi: 10.1091/mbc.E12-03-0194

Melamed, D., Pnueli, L., and Arava, Y. (2008). Yeast translational response to high salinity: global analysis reveals regulation at multiple levels. RNA 14, 1337-1351. doi: 10.1261/rna.864908

Miller, S. B. M., Ho, C., Winkler, J., Khokhrina, M., Neuner, A., Mohamed, M. Y. H., et al. (2015). Compartment-specific aggregases direct distinct nuclear and cytoplasmic aggregate deposition. EMBO J. 34, 778-797. doi: 10.15252/embj.201489524

Nguyen, T. T. M., Iwaki, A., and Izawa, S. (2015). The ADH7 promoter of Saccharomyces cerevisiae is vanillin-inducible and enables mRNA translation under severe vanillin stress. Front. Microbiol. 6:1390. doi: 10.3389/fmicb.2015.01390

Nguyen, T. T. M., Kitajima, S., and Izawa, S. (2014). Importance of glucose-6phosphate dehydrogenase (G6PDH) for vanillin tolerance in Saccharomyces cerevisiae. J. Biosci. Bioeng. 118, 263-269. doi: 10.1016/j.jbiosc.2014. 02.025

Obrig, T. G., Culp, W. J., McKeehan, W. L., and Hardesty, B. (1971). The mechanism by which cycloheximide and related glutarimide antibiotics inhibit peptide synthesis on reticulocyte ribosomes. J. Biol. Chem. 246, 174-181.

Schmitt, M. E., Brown, T. A., and Trumpower, B. L. (1990). A rapid and simple method for preparation of RNA from Saccharomyces cerevisiae. Nucleic Acids Res. 18, 3091-3092. doi: 10.1093/nar/18.10.3091

Sikorski, R. S., and Hieter, P. (1989). A system of shuttle vectors and yeast host strains designed for efficient manipulation of DNA in Saccharomyces cerevisiae. Genetics 122, 19-27.

Slater, M. R., and Craig, E. A. (1987). Transcriptional regulation of an hsp70 heat shock gene in the yeast Saccharomyces cerevisiae. Mol. Cell. Biol. 7, 1906-1916. doi: 10.1128/MCB.7.5.1906

Sopko, R., Huang, D., Preston, N., Chua, G., Papp, B., Kafadar, K., et al. (2006). Mapping pathways and phenotypes by systematic gene overexpression. Mol. Cell 21, 319-330. doi: 10.1016/j.molcel.2005.12.011

Takahashi, T., Satake, S., Hirose, K., Hwang, G. H., and Naganuma, A. (2011). A screening for essential cell growth-related genes involved in arsenite toxicity in Saccharomyces cerevisiae. J. Toxicol. Sci. 36, 859-861. doi: 10.2131/jts. 36.859

Takahashi, T., Shimoi, H., and Ito, K. (2001). Identification of genes required for growth under ethanol stress using transposon mutagenesis in Saccharomyces cerevisiae. Mol. Genet. Genomics 265, 1112-1119. doi: 10.1007/s0043801 00510

Takemura, R., Inoue, Y., and Izawa, S. (2004). Stress response in yeast mRNA export factor: reversible changes in Rat8p localization are caused by ethanol stress but not heat shock. J. Cell Sci. 117, 4189-4197. doi: 10.1242/jcs.01296

Teixeira, D., Sheth, U., Valencia-Sanchez, M. A., Brengues, M., and Parker, R. (2005). Processing bodies require RNA for assembly and contain nontranslating mRNAs. RNA 11, 371-382. doi: 10.1261/rna.7258505

Teixeira, M. C., Raposo, L. R., Mira, N. P., Lourenço, A. B., and Sá-Correia, I. (2009). Genome-wide identification of Saccharomyces cerevisiae genes required for maximal tolerance to ethanol. Appl. Environ. Microbiol. 75, 5761-5772. doi: 10.1128/AEM.00845-09

Uesono, Y., and Toh-E, A. (2002). Transient inhibition of translation initiation by osmotic stress. J. Biol. Chem. 277, 13848-13855. doi: 10.1074/jbc.M108848200

van Voorst, F., Houghton-Larsen, J., Jønson, L., Kielland-Brandt, M. C., and Brandt, A. (2006). Genome-wide identification of genes required for growth of Saccharomyces cerevisiae under ethanol stress. Yeast 23, 351-359. doi: 10.1002/yea.1359

Vera, M., Pani, B., Griffiths, L. A., Muchardt, C., Abbott, C. M., Singer, R. H., et al. (2014). The translation elongation factor eEF1Al couples transcription to translation during heat shock response. eLife 3:e03164. doi: 10.75554/eLife. 03164

Yamamoto, A., Mizukami, Y., and Sakurai, H. (2005). Identification of a novel class of target genes and a novel type of binding sequence of heat shock transcription factor in Saccharomyces cerevisiae. J. Biol. Chem. 280, 1191111919. doi: $10.1074 /$ jbc.M411256200

Yamamoto, Y., and Izawa, S. (2013). Adaptive response in stress granule formation and bulk translational repression upon a combined stress of mild heat shock 
and mild ethanol stress in yeast. Genes Cells 18, 974-984. doi: 10.1111/gtc. 12090

Yang, J., Bae, J. Y., Lee, Y. M., Kwon, H., Moon, H. Y., Kang, H. A., et al. (2011). Construction of Saccharomyces cerevisiae strains with enhanced ethanol tolerance by mutagenesis of the TATA-binding protein gene and identification of novel genes associated with ethanol tolerance. Biotechnol. Bioeng. 108, 1776-1787. doi: 10.1002/bit.23141

Zid, B. M., and O'Shea, E. K. (2014). Promoter sequences direct cytoplasmic localization and translation of mRNAs during starvation in yeast. Nature 514, 117-121. doi: 10.1038/nature13578
Conflict of Interest Statement: The authors declare that the research was conducted in the absence of any commercial or financial relationships that could be construed as a potential conflict of interest.

Copyright () 2016 Yamauchi and Izawa. This is an open-access article distributed under the terms of the Creative Commons Attribution License (CC BY). The use, distribution or reproduction in other forums is permitted, provided the original author(s) or licensor are credited and that the original publication in this journal is cited, in accordance with accepted academic practice. No use, distribution or reproduction is permitted which does not comply with these terms. 